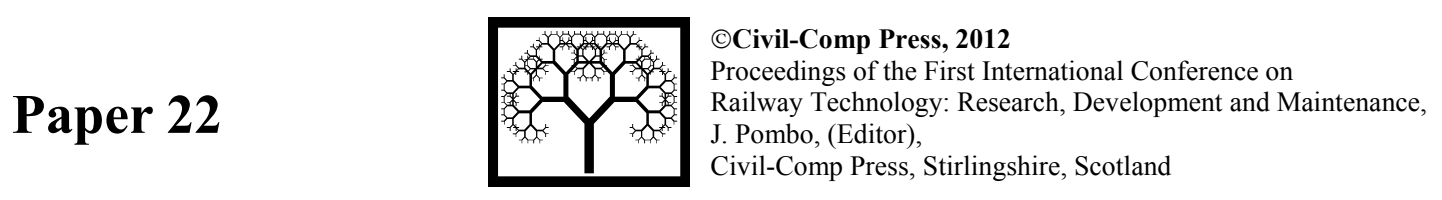

\title{
Novel Rail Vehicle Concepts for a High Speed Train: The Next Generation Train
}

\author{
J. Winter \\ Institute of Vehicle Concepts \\ German Aerospace Center (DLR), Stuttgart, Germany
}

\begin{abstract}
It is well known that the German Aerospace Center is researching in the areas aeronautics and space and it is the German Space Agency. Less known is that for about ten years it has been researching in the areas of transport and energy as well. This paper gives an overview concerning one of the major transport research projects of the German Research Center.
\end{abstract}

Keywords: transport, high-speed train, next generation train, multiple-unit.

\section{Introduction}

Due to growing globalization and worldwide mobility the total "transport" becomes more and more complex. In the area of railways especially, the requirements for interoperability of cross-border transport is becoming more challenging partly because of quite different national requirements.

The specification put forward to the rail vehicle manufacturers have massively changed with the transfer from product driven to project driven business: in former times a series was produced over the years without major changes. Today a manufacturer has a competitive advantage if he can quickly deliver a tailored product which is more efficient and which saves resources.

Furthermore manufacturers see themselves exposed to rapid price deterioration, smaller lots and at the same time rising demands for safety and efficiency.

Significant drivers of this evolution are:

- progressively rising energy costs

- growing demand for reduced emissions

- growing importance of life cycle costs of a vehicle compared to the investment costs 
- strong requirements for safety of future vehicles

- competitiveness with other means of transport (especially aircraft)

- rising expectations of passengers on vehicle comfort

The topics of emission reduction and safety have a high social relevance.

\section{Project Next Generation Train - NGT}

\subsection{State of the project}

The German Aerospace Center - Deutsches Zentrum für Luft- und Raumfahrt (DLR) project Next Generation Train - NGT [1], has since 2007, delivered valuable results to maintain the existing advance in rail transport compared to other modes of transport. These results are presented in this paper in a comprehensive way. More detailed the results are given in [2].

In the NGT framework the DLR bundles its skills along the research value added chain. The focus is thus clearly on the rail vehicle. The interfaces with track, signalling and power supply are treated in a complementary way.

The project has been reviewed every 5 years and monitored in between by the Helmholtz Association. The Helmholtz Association is a community of 18 scientifictechnical and biological-medical research centres. The last review was in 2008 .

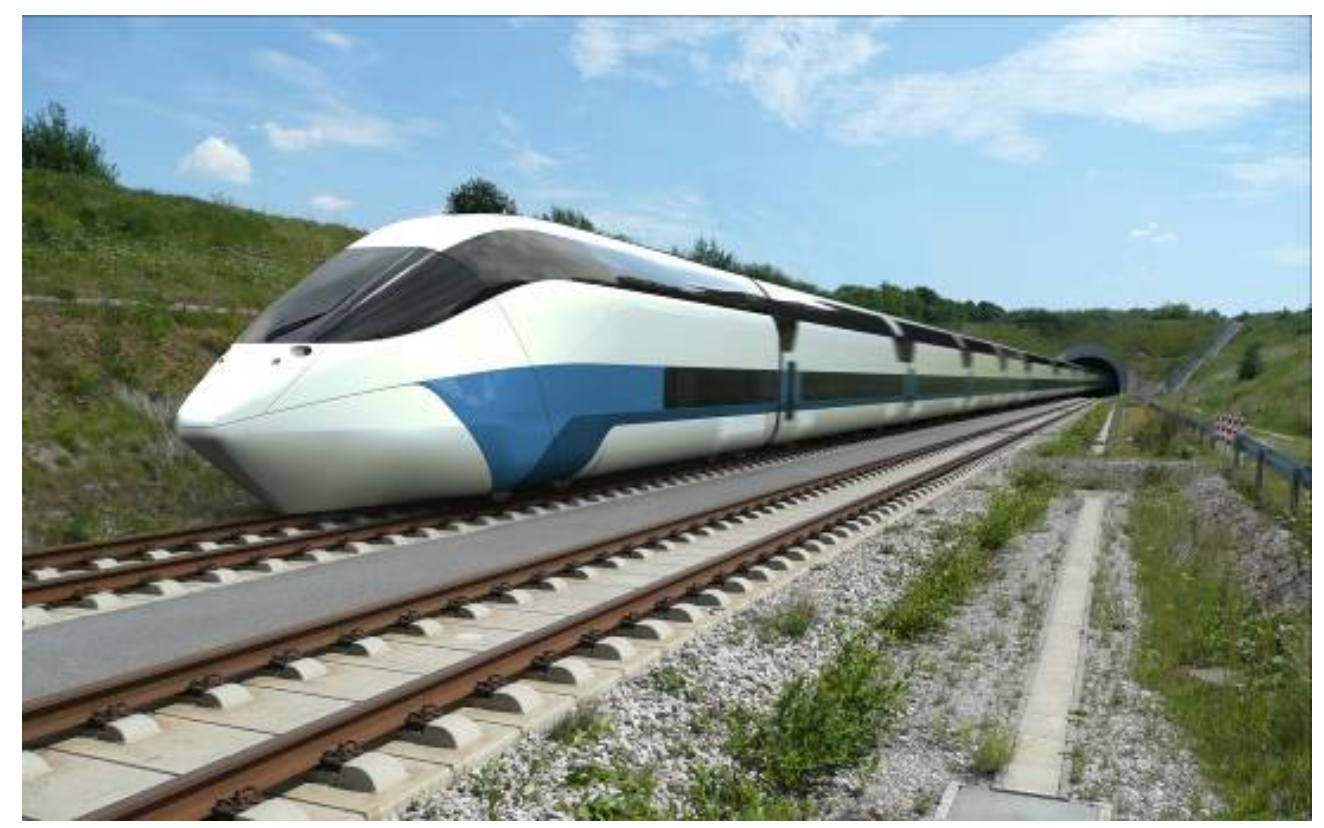

Figure 1: NGT HST running on a high speed line in 2035 (artist's impression) 
The project Next Generation Train has in

- Phase I (2007 - 2009) mainly developed the rail vehicle concept shown in Figure 1. This was defined by collecting customer requirements, developing the functional requirements specification and defining the high-speed transport NGT HST in a design specification as a double-decker electrical multiple-unit (EMU) motor car train running with an operational speed of $400 \mathrm{~km} / \mathrm{h}$. Benchmark is the Deutsche Bahn InterCity-Express ICE3 high-speed train.

- Phase IIa (2010 - 2013) will complete the rail vehicle concept NGT HST by defining propulsion and braking concepts. A middle-car is under detailed construction at this time. The sub-assemblies are to be tested in 2013.

Furthermore, the research results for the high-speed train are adapted into an intercity type NGT REGIO intended to feed the high-speed service.

- Phase IIb (2011 - 2015) to design the NGT REGIO as a double-decker multipleunit motor car train with hybrid propulsion for electrified and non-electrified lines. The operational speed is $230 \mathrm{~km} / \mathrm{h}$. The passenger flow and an overall crash-concept are under investigation.

Sections of the middle-car will be shown at the InnoTrans 2012 exhibition in Berlin. On display will be a section (size 1:1) of the light weight construction of the passenger module and of the wheel set module including a mechatronic single wheel single running gear mock-up.

Also under construction is a size 1:1 generic laboratory representing a middle-car. The generic laboratory is mainly a standard room in which to investigate the performance of air conditioning systems on behalf of the railway industry.

In addition the DLR bought a middle car of a Bombardier Transportation Talent2 train to enable the DLR to investigate realistically the thermal as well as the noise insulation of car bodies.

In October 2010 the DLR finished the development of the concept and construction of a unique wind tunnel to investigate head pressure wave in a tunnel and crosswind effects on high-speed trains. Vehicle models can vary between 1:20 and 1:100 with maximum mass of $10 \mathrm{~kg}$.

In the same year the DLR built a laboratory to investigate passenger comfort.

Further large scale research equipment is proposed, as for example a complete research train. 


\subsection{Project goals}

\subsubsection{Research objectives}

The general objectives of the passenger NGT HST project are:

1. performance and attractiveness of rail vehicle increased by

- scheduled operational speeds as high as reasonable

- reduced travel time for passengers within the zone of influence of an HSL

- large passenger/load capacity

- outstanding passenger comfort (noise, vibration, pressure waves, air conditioning, baggage handling, seat access, information, entertainment...)

- flexible, modular vehicle platform with common parts as far as possible

- strictly lightweight design

2. safety and reliability of rail vehicle increased by

- car body structure optimised for relevant crash scenarios

- mechatronic system solutions (running gear, brakes,....) optimised

- aerodynamic resistance reduced by about $25 \%$ (compared to ICE2)

- design of the running gear and safe passenger compartments (tilt resistance, derailment at curve entry,...)

- reduced wear at wheel/rail contact

3. energy efficiency increased by

- specific energy consumption reduced by $50 \%$ compared to ICE3 at $300 \mathrm{~km} / \mathrm{h}$

- larger number of passenger seats

- aerodynamic optimization of the rail vehicle

- intelligent energy management of all consumers onboard the train

\subsubsection{Benefits}

Added value is generated by the NGT project especially by a holistic view of all issues and the synergy with related research in the fields of cars and aerospace. In the sense of "one" DLR very good networking has been established between the DLR Institutes. The work of specialized scientists is always coordinated by systems engineers via specifications and discussions in the form of workshops. The project bundles together the competences of DLR in the area of rail vehicles along the value added chain of research and aims for hands-on research results as, for example, in development of components.

As the DLR will never build rail vehicles it is very important for the NGT project to work in close cooperation with the railway industry and the railway operators. The results are provided for product development even during the run time of the project whenever this is reasonable. 


\section{Compilation of results}

The project NGT gets results which, on the one hand, are independent of a specific vehicle and on the other hand vehicle specific, as for example for the high-speed electrical multiple-unit motor car train NGT HST. The results of a more general nature will be applied to a number of rail vehicle types.

\subsection{Rail vehicle concept}

The priority is high operational flexibility of the NGT. To assist easy maintenance and the replacement of defective cars, the single car principle was selected. Each car can be shunted separately, which is a considerable advantage for train composition and maintenance. A double-decker train set of eight middle cars and two end cars is formed over the whole length of $202 \mathrm{~m}$ (see Figure 2). Several train sets can be coupled by means of an optical coupling.

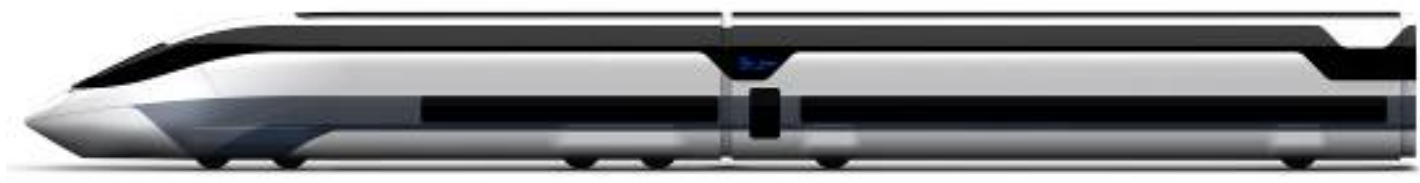

Figure 2: Side view showing the $21 \mathrm{~m}$ end car and a $20 \mathrm{~m}$ middle car

The operational flexibility is further increased by the possibility to split train sets dynamically into directional trains, i.e. the train sets can be optically coupled and uncoupled during the run. After introducing the flexible block intervals as a principle of train safety it will be possible to increase the traffic flow on the lines considerably.

\subsection{Passenger flow concept}

Based on simulations with the traffic oriented microscopic simulator (TOMICS), a DLR software package for modelling individual movement of persons in any chosen traffic environment, and the boarding and disembarking times of passengers was analysed.

The NGT HST cars have through corridors on both levels (see Figure 3). This means that there are no stairs inside the cars.

Passenger baggage is dealt with separately in the end car by a baggage handling system. 


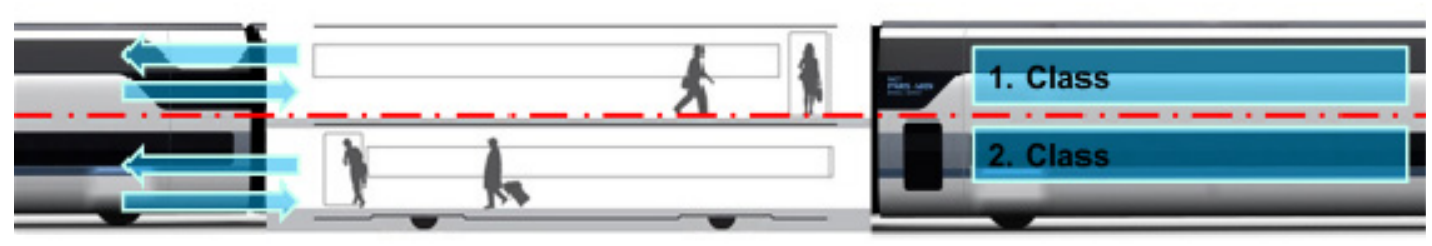

Figure 3: Level concept of the high-speed train

The best results were for a double-decker train set with horizontal class divisions with up to 790 passengers in two classes, an onboard restaurant and an area for people with reduced mobility. The passengers can board at either level and disembark through the diagonally opposite car doors (see Figure 4). The car doors are $1 \mathrm{~m}$ wide. The upper level could be reached by passenger bridges, stairs or with other architectural solutions.

A guidance system ensures that passengers select the optimum entrance.
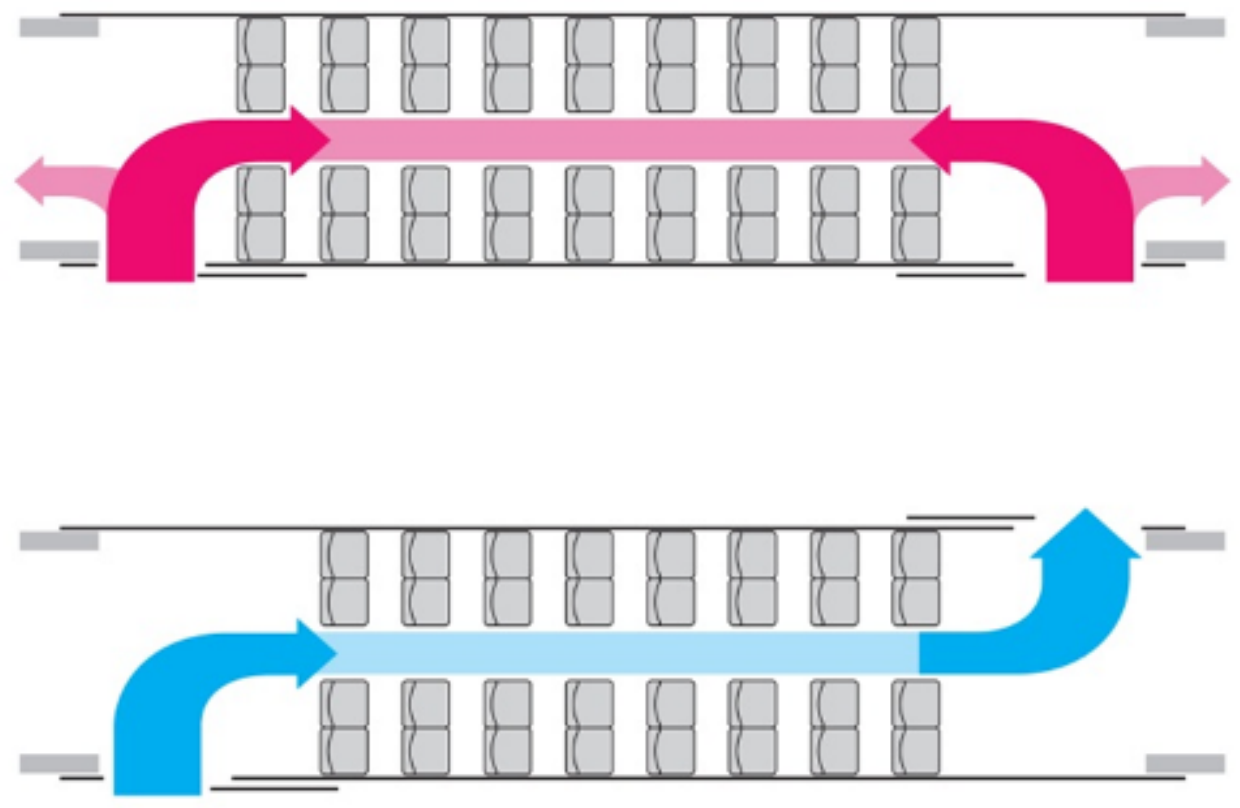

Figure 4: Passenger flow today in red and with the new concept below in blue 


\subsection{Propulsion and braking concept}

The power supply is assumed to be in the track in the future, which means that the maintenance intensive overhead line with its catenary system can be dispensed with. The propulsion concept accordingly proposes a contactless power pick-up from the rail track distributed along the length of the train set. The challenge of developing the components required for this is being worked on. From the vehicle point of view the noisy, heavily wearing pantograph will no longer be required.

The end cars provide $50 \%$ of the propulsion power of about $18 \mathrm{MW}$ through the synchronous motors of the single wheel double running gear, the remaining propulsion power is provided by the highly integrated wheel motors of the single wheel single running gear. As a result, the train set has above average acceleration. The double-decker high speed train set has a scheduled speed of $400 \mathrm{~km} / \mathrm{h}$ and is approved up to $440 \mathrm{~km} / \mathrm{h}$.

The braking concept provides for the use of different brakes depending on the speed. It is principally run with anticipative driver assistance and on coasting. Should this not suffice, regenerative brakes operating wear-free and linear eddy current brakes are used to slow down the train. At top speed aerodynamic brakes are activated additionally. At lower speeds, linear eddy current brakes can be used above $50 \mathrm{~km} / \mathrm{h}$. The operational braking distance at $440 \mathrm{~km} / \mathrm{h}$, in contrast to that in the TSI RST [3] which requires $21 \mathrm{~km}$, can be reduced to $10 \mathrm{~km}$. This in comparison to the ICE3 with a $4 \mathrm{~km}$ braking distance (at $300 \mathrm{~km} / \mathrm{h}$ ) is still a lot.

For emergency braking mechanical brakes are activated in addition. The braking distance is then $6.4 \mathrm{~km}$.

\subsection{Mechatronic track-guidance for single-wheel single running gear}

The wear and the noise generation of the running gear can be reduced by a mechatronic wheel set as a radial controllable differential powered single wheel single running gear (or double running gear), which steers the wheel discs actively in bends (Figure 5).

In comparison with the conventional wheel set, its passive self-centring mechanism has to be sacrificed for this advantage. This function can, for example, be taken over by an active guidance concept with a robust sensor. 


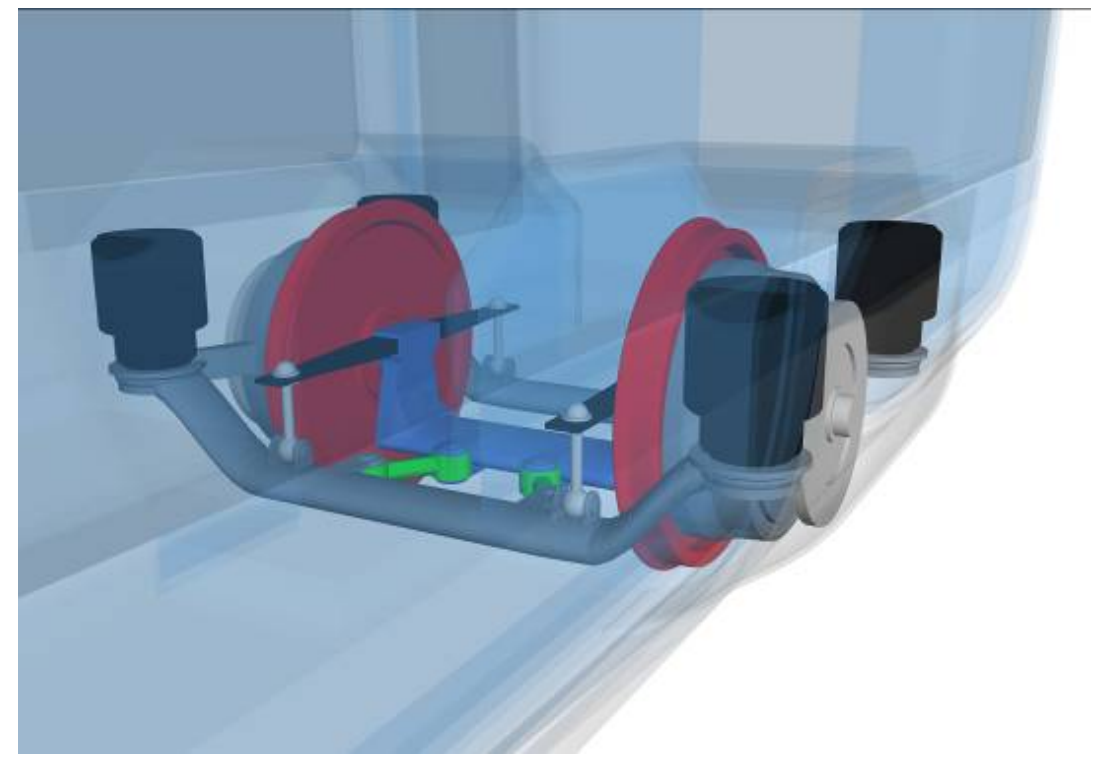

Figure 5: Single-wheel single running gear set

With the aid of the moment of force sensors integrated in the wheel linkage the position of the running gear on the rail is indirectly detected. The actuators (permanent synchronous motors) and the other sensors (transmitter, temperature sensor) are built into every wheel. The wheel pairs have a degree of freedom around the vertical axis and can be adjusted in bends so that the slip which occurs and therefore also the wear can be reduced. In addition, through the speed decoupling of the wheels also in very tight bends both the longitudinal and the lateral slip can be reduced, which means that the conditions for squeaking in bends are no longer there and noise reduction is achieved.

The mechatronic track-guidance, together with dynamic comfort design, which also includes the dynamic link to the adjacent car, more than compensate for the potential dynamic disadvantages of single axle running gear in comparison to bogies.

\subsection{Innovative car body lightweight construction}

In a train set with single cars the car bodies utilise the theoretically available space to an optimum. The span width is reduced in the same car body length in comparison to an articulated set in a bridge car - Jacobs - layout and from the overhangs an opposite moment results in a deflection. This means that, in concept, a weight reduction has already been achieved.

The chosen concept with only two single running gear per car requires considerable efforts in lightweight construction as the permitted maximum axle load is $16 \mathrm{t}$ (TSI RST [3] 17 tonnes) and the vehicle weight is limited to 32 tonnes. Required power 
savings when accelerating, when at constant speed and when breaking, reduction of the superstructure load or damage and reduction in environmental pollution by reducing emissions, are further substantial reasons for strictly lightweight construction.

The train set concept uses a mixture of integral and modular product architecture in order, given the substantial product differentiation, to be able to manufacture cost effectively using a large number of the same components and the usually small batches required (Figure 6).

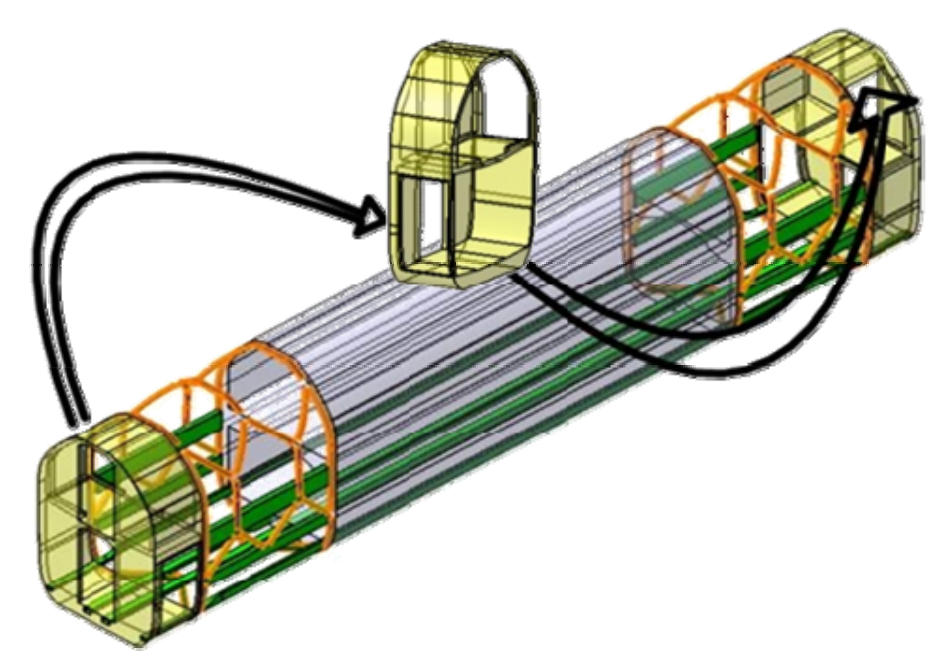

Figure 6: Construction modules of a middle car

To fully exploit the maximum lightweight construction potential, the fundamental principle of multi-material design is followed, where the respective materials are used where their optimum potential can be best realised. In particular fibre reinforced plastics and light alloys are used to achieve the low structural weights. The modular DLR concept consists of a framework structure with load bearing infills in fibre reinforced lightweight construction, which divides the car bodies with defined ribs in various parallel finished sections.

The objective is to achieve a weight reduction of up to $30 \%$ in comparison to metal construction by systematic use of multi-material design philosophy and the novel $\mathrm{rib} /$ panel method of construction.

As much functionality as possible is integrated into the car body structure in order to best utilise the interior space volumes which are limited by the G2 clearance. The largest components are the radial controllable differential powered single wheel single running gear. 


\subsection{Aerodynamics}

The compound topic of aerodynamics touches on aspects of the vehicle, the infrastructure and the operation and is therefore very complex. With the increasing scheduled speed of trains, actual effects have occurred time and again where it is not possible to simulate their dynamic movement and aerodynamic relationships with the available mathematical methods. Results from models often could not be verified in the wind tunnels. Here the project is performing pioneering work.

For example, can the frontal wave of the train set be reduced by a larger thickness ratio. For the NGT a medium thickness ratio of the front is used because, in future, tunnels on high speed lines will be built as single tube, but with an intake funnel to continuously displace air.

The immunity to crosswinds is sustainably ensured despite the light end and middle cars of the NGT train set by active control elements.

To investigate both phenomena, DLR has since 2010 had a worldwide unique wind tunnel (Figure 7).

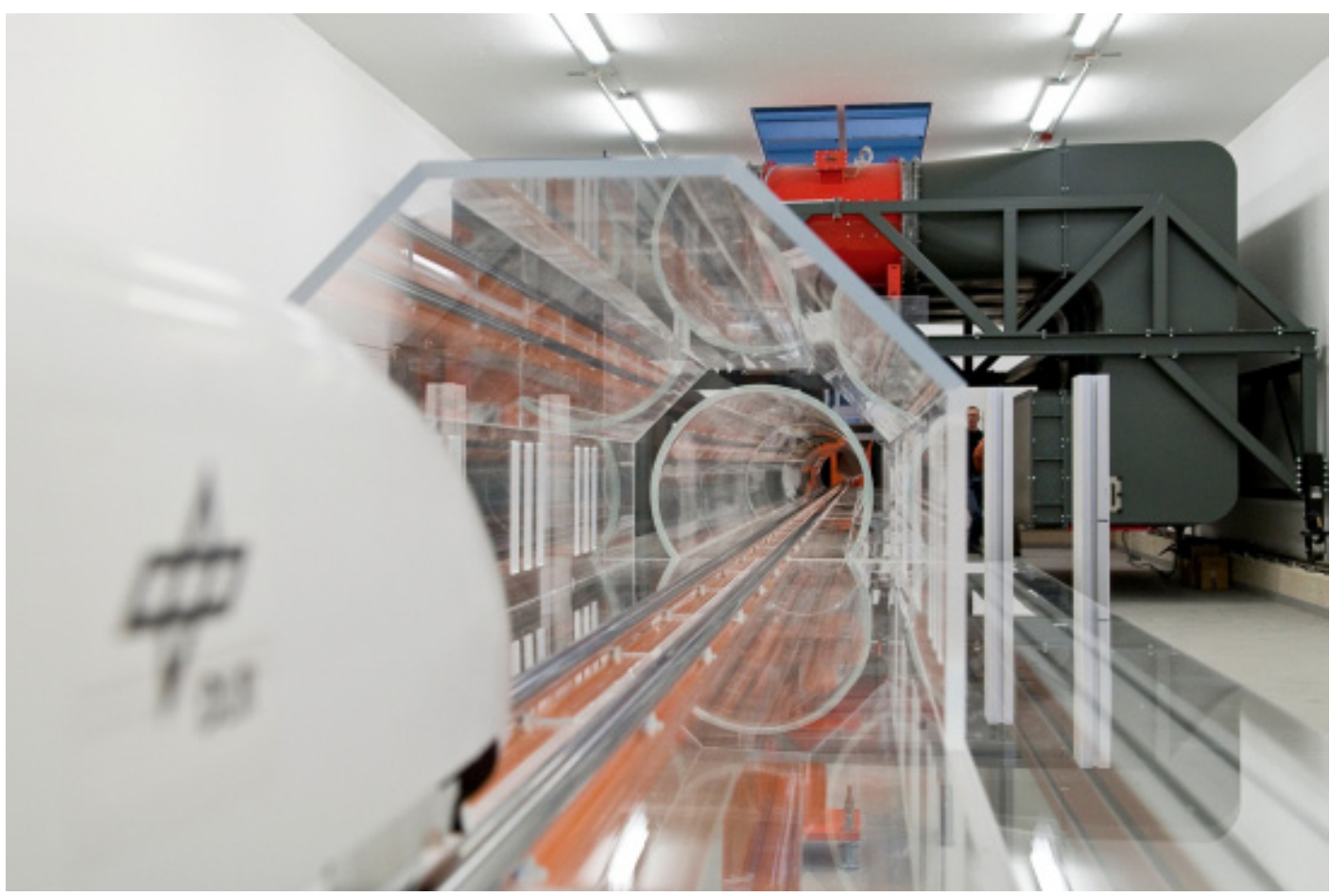

Figure 7: Tunnel and crosswind research facility

The aerodynamic shape of the NGT is optimised, for example, by no pantographs, seamless car platforms and abandonment of air cooled brake discs. Thus, at the same time the noise generated by vortices is reduced significantly. 


\subsection{Industrial design}

The outside design shown in Figure 8 was designed in close relationship to the aerodynamic researches of the DLR, the specific construction methods, the optimum people/machine interface and the aesthetic external effect, architecture and environment.

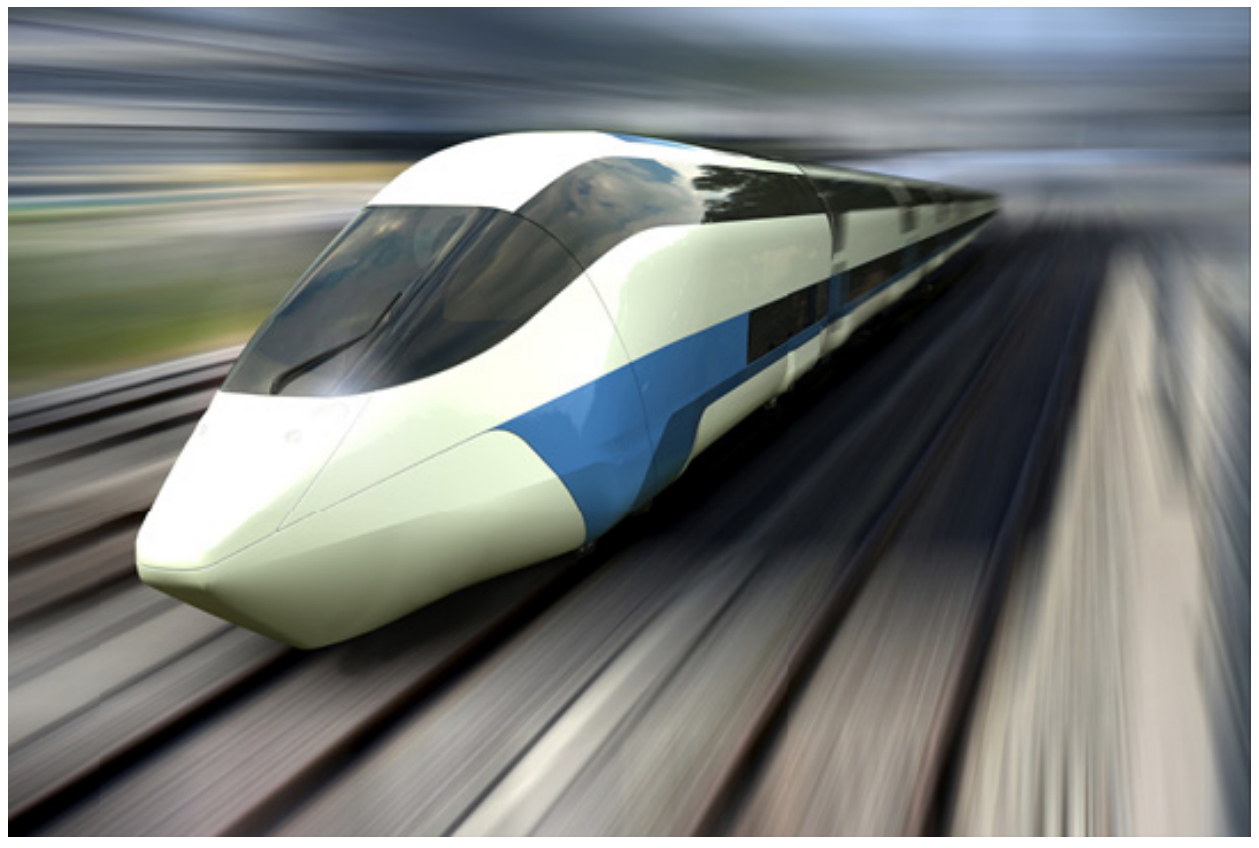

Figure 8: NGT NST running at maximum speed $460 \mathrm{~km} / \mathrm{h}$ (artist's impression)

In the interior area different passenger groups and travel requirements and variable modular concepts were developed and demonstrated. Particular focuses are on the boarding and disembarking areas as the "visiting card" of the Next Generation Train.

Of the middle cars of the NGT there are seven seating cars which because of the headroom take first class on the upper deck and second class on the lower. The accesses are arranged at both levels. A change of levels is possible via a lift with disabled access in the restaurant car, which is located in the middle of the train set. This has the restaurant on the upper level and on the lower level are special compartments for parents and children and passengers with reduced mobility.

The bright light and generous fenestration design as well as novel stowage possibilities for hand luggage offers a new level of rail vehicle passenger comfort.

\subsection{Creating a high-speed railway network}

We have already developed software tools enabling us to calculate for a given country or continent the potential passenger volume for the high speed line to be 
built, the estimated construction costs for new high speed lines and the operational schedule for the traffic (including use of already existing slow track sections). This gives us an estimate of the total investment.

In addition we have tools for calculating the energy consumption on certain lines or for a given operational schedule as well as the other Live Cycle Costing (LCC).

Furthermore we can take care of the passenger flow in the train and through the station. Thus, we can plan railway stations and rail vehicle concepts to given optimal performance.

We can advise on how to create a high speed rail network that makes sense - in cases where we have access to a sound data base. For Europe the software tools are verified by the existing Trans-European Network - TEN planning of the European Commission.

\subsection{Modelling of life cycle costs}

Based on the high investment costs and the long service life, the life cycle costs in introducing innovative rail vehicles to the market play an important role. Savings in maintenance and repair costs cancel out higher purchase prices in many cases after a short period of time. A reliable method of quantifying product costs over the total life is provided by LCC analysis. This enables economic advantages of new technologies to be compared to conventional vehicles.

The comparison of the energy costs for the operation of the NGT and of a reference vehicle can be carried out, for example, on the basis of the UIC High Speed Service Profile (at $300 \mathrm{~km} / \mathrm{h}$ ). The costs here are based on starting operation in 2020 and with a service life of the vehicle of 30 years. In calculating the overall costs, an average annual inflation rate of $1.8 \%$ was taken into account. The $27 \%$ lower power consumption per train kilometre means that over the whole service life an average saving of approximately $500.000 €$ per annum can be achieved. On a per seat basis this there is a $50 \%$ energy saving per seat, which was specified as a design objective.

Preliminary calculations have shown that due to the innovative technologies, employed in the NGT, although the investment costs are increased, the operating costs can, however, also be reduced. As a result the NGT succeeds in achieving cost savings over the whole life cycle in comparison to conventional rail vehicles. It will only be possible to have quantitative data towards the end of the project term in 2013. 


\section{Conclusions}

The next generation train (NGT) of the DLR is characterised by comparatively low energy consumption, low noise generation, pleasant air conditioning, optimised passenger flows, a future-orientated exterior and interior design and low wear on wheel to rail contact. The Deutsche Bahn InterCity Express - the ICE3 high-speed train is a benchmark for the achievement of the research objectives.

\section{References}

[1] http:/www.dlr.de/dlr/en/desktopdefault.aspx/tabid-10467/740_read-916/; 05.02 .2012

[2] J. Winter (Editor), "NGT - Next Generation Train", DVV Media Group / eurailpress, RTR Special 2011

[3] Trans-European High-speed Rail System, Technical Specification for Interoperability "Rolling Stock" - TSI RST, Official Journal of the European Union L84/132 from 21.02.2008 